\title{
The Effectiveness of Dexmedetomidine in Vacuum-Assisted Breast Biopsy Under Monitored Anesthesia Care
}

\author{
Kyung Woo Kim ${ }^{1}$, Jun Ha Park ${ }^{1}$, Seunghwan Kim ${ }^{1}$, Eun Jin Ahn ${ }^{1}$, Hyo Jin Kim ${ }^{1}$, Hey Ran Choi ${ }^{1}$, Yeo Goo Chang ${ }^{2}$, \\ Si Ra Bang ${ }^{1}$ \\ ${ }^{1}$ Department of Anesthesiology and Pain Medicine, Inje University Seoul Paik Hospital, Seoul, Korea \\ ${ }^{2}$ Department of General Surgery, Inje University Seoul Paik Hospital, Seoul, Korea
}

Objectives: Vacuum-assisted breast biopsy (VABB) is a widely used technique for the diagnosis of breast lesions. It is carried out with local anesthesia, but procedural pain and stress are still problematic. Dexmedetomidine is a $\alpha-2$ receptor agonist that can sedate without significant respiratory depression. The study aimed to report the effectiveness of sedation with monitored anesthesia care (MAC) using dexmedetomidine in VABB.

Methods: This was a retrospective chart review of patients who received VABB under MAC with dexmedetomidine. Forty-seven patients during the period of February 2015 to July 2016 were included. We collected data on patient characteristics, infusion drug and dose, induction to incision time, anesthetic, operation, and recovery time and other complications and vital signs.

Results: The mean operating time was $50.1 \pm 24.9$ minutes, and the anesthetic time was $71.2 \pm 28.3$ minutes. The mean time from induction to incision was $17.0 \pm 5.2$ minutes, and the recovery time was $20.1 \pm 10.3$ minutes. None of the patients needed an advanced airway management. Further, none of them showed hemodynamic instability.

Conclusions: VABB was successfully performed with MAC using dexmedetomidine, and there was no respiratory depression or hemodynamic instability.

Key Words: Dexmedetomidine, Mammotome, Monitored anesthesia care, Vacuum-assisted breast biopsy

Vacuum-assisted breast biopsy (VABB; Mammotome ${ }^{\circledR}$ ) is a minimally invasive method usually performed under ultrasound guidance. It has a smaller incision, less blood loss, a shorter recovery time, and a shorter operative time than excisional surgery. ${ }^{1}$ Conventionally, VABB is performed under local anesthesia using an 8-14 gauge needle in outpatient settings, ${ }^{1,2}$ however, procedure dura- tion, large needle diameter, pathologic diagnosis, blood loss, and the associated pain ${ }^{3,4}$ can be physical and mental stressors to patients. ${ }^{5,6}$

Monitored anesthesia care (MAC) is a diagnostic or therapeutic procedure performed under the supervision of an anesthesiologist to reduce residual sedation through appropriate pain relief and for a quick discharge. It has been described
Corresponding Author: Si Ra Bang, Department of Anesthesiology and Pain Medicine, Inje Uniersity Seoul Paik Hospital, 9, Marunnai-ro, Jung-gu, Seoul 04551, Korea

Tel: +82-2-2270-0094 Fax: +82-2-2270-0094 E-mail: sira1045@naver.com

\section{(c) (1) (2)}

Articles published in Kosin Medical Journal are open-access, distributed under the terms of the Creative Commons Attribution Non-Commercial License (http://creativecommons.org/licenses/by-nc/4.0/) which permits unrestricted non-commercial use, distribution, and reproduction in any medium, provided the original work is properly cited.
Received: Nov. 19, 2018

Revised: Jan. 08, 2019

Accepted: Jan. 16, 2019 
by the American Society of Anesthesiologists as a specific anesthesia service for diagnostic or therapeutic procedures performed under local anesthesia along with sedation and analgesia, titrated to a level that preserves spontaneous breathing and airway reflexes. ${ }^{7}$ MAC is useful for daytime surgery because it is faster than general anesthesia and relieves the anxiety, boredom, and tension patients feel during local anesthesia. ${ }^{8}$ However, because of the combined use of sedatives and analgesics, respiratory depression can often occur during MAC.

Dexmedetomidine (Precedex ${ }^{\circledR}$, Hospira, Lake Forest, IL, USA) is a centrally acting $\alpha-2$ receptor agonist. It can sedate at desired depths without significant respiratory depression and with analgesic action. ${ }^{9,10}$ In addition, dexmedetomidine has a sympatholytic effect that can attenuate the stress response to surgery, mitigating tachycardia and hypertension. ${ }^{11}$ Therefore, it is useful for MAC. Here, we report the effectiveness of VABB under MAC. Specifically, we evaluated sedation using dexmedetomidine MAC in VABB.

\section{MATERIALS AND METHODS}

\section{Methods}

This was a retrospective study conducted following approval from the institutional review board of our center. From February 2015 to July 2016, we selected patients who underwent VABB using MAC with dexmedetomidine and ultimately enrolled 47 patients. We excluded patients who were taking sedatives or opioid medication or who were undergoing multiple operations.

$\mathrm{VABB}$ was performed using an 8-gauge vacuum probe (Mammotome ${ }^{\circledR}$ Breast Biopsy system/ Ethicon Endo-Surgery In., a Johnson \& Johnson Co., Cincinnati, OH, USA). All procedures were performed by one surgeon. Noninvasive blood pressure (BP), oxygen saturation, and ECG were monitored. MAC was induced with dexmedetomidine until the patient stopped responding to voice and pain. The surgeon performed the local infiltration of the insertion sites for the probes with $2 \%$ lidocaine with a total volume of 5-10 $\mathrm{ml}$. With the patient in the supine position, the surgeon performed the VABB under ultrasound guidance, inserting an 8-gauge Mammotome ${ }^{\circledR}$ probe through a small skin incision. After the procedure, the biopsy site was compressed, and chest wrapping bandage was applied.

We collected our data retrospectively from the patients' medical charts, specifically on the following variables: age, height, weight, underlying disease, number of breast biopsies, premedication drugs and doses, dexmedetomidine loading, infusion and total dose, other analgesics or sedatives, induction to incision time (the time from start of dexmedetomidine until there was no response to voice and pain), total anesthetic and operation time, recovery time (the time from discontinuation of dexmedetomidine to eye opening), complication (nausea, vomiting, bleeding), vital signs (BP, heart rate, pulse oximetry sat- 
uration), and bispectral index (BIS), which represents sedation level. We defined clinically significant hypotension (Systolic BP $<90 \mathrm{mmHg}$, Mean BP < $60 \mathrm{mmHg}$ ) or clinically significant bradycardia (Heart rate $<50$ and 20\% decrease in heart rate compared to the preanesthetic rate).

\section{Statistical analysis}

This study was descriptive, and we did not test a hypotheses or calculate sample sizes. Data are presented as mean (standard deviation, SD). The vital signs were analyzed by repeated measures ANOVA. All the statistical analyses were performed using the MedCalc software package, version 17.9 (MedCalc Software, Ostend, Belgium) for Windows ${ }^{\circledR}$.

\section{RESULTS}

In total, we screened 49 patients and enrolled 47 into the study; we excluded one patient who had undergone other procedures and one who had been treated with an additional dose of dexmedetomidine due to reoperation. The patients' demographic data and dosage information are shown in Table 1. The operating time was 50.1 \pm 24.9 minutes, and the anesthetic time was 71.2 \pm 28.3 minutes. The mean time from the administration of dexmedetomidine to the start of the procedure was $17.0 \pm 5.2$ minutes, and the time to recovery after discontinuation of dexmedetomidine was $20.1 \pm 10.3$ minutes on an average. The dosage information of dexmedetomidine and rescue drug are shown in Table 2 . The total dexmedetomidine dose was $101.1 \pm 34.1 \mu \mathrm{g}$.

In the recovery room, one patient received atropine $0.25 \mathrm{mg}$ due to bradycardia, and one received ramosetron $0.3 \mathrm{mg}$ due to nausea. Systolic and diastolic BP and heart rate were different at the end of procedure compared with the initial $\mathrm{BP}$ and heart rate. However, on measuring vital signs, none of the patients had clinically significant hypotension or clinically significant bradycardia (Table 3). No patient needed an advanced airway because of the decreased oxygen saturation during the procedures.

Table 1. Baseline patient characteristics

\begin{tabular}{lc}
\hline & $\mathrm{n}=47$ \\
\hline Age $(\mathrm{yr})$ & $41.9 \pm 9.2$ \\
Height $(\mathrm{cm})$ & $161.0 \pm 6.3$ \\
Weight $(\mathrm{kg})$ & $60.7 \pm 9.1$ \\
Hypertension & 3 \\
Hypothyroidism & 1 \\
Number of biopsy $(\mathrm{n})$ & $2.4 \pm 1.3$ (both breasts, $\mathrm{n}=22$ ) \\
\hline
\end{tabular}

Values are expressed as mean $\pm \mathrm{SD}$ 
Table 2. The dosage information.

\begin{tabular}{cc}
\hline Drugs & Dose \\
\hline Total dexmedetomidine dose $(\mu \mathrm{g})$ & $101.1 \pm 34.1$ \\
Dexmedetomidine loading dose $(\mu \mathrm{g} / \mathrm{kg})$ for 10 minutes & $0.9 \pm 0.1$ \\
Dexmedetomidine infusion dose $(\mu \mathrm{g} / \mathrm{kg} / \mathrm{hr})$ & $0.4-1.0$ \\
Rescue midazolam $(\mathrm{mg})$ & $1.4 \pm 0.7, \mathrm{n}=28$ \\
Rescue fentanyl $(\mu \mathrm{g})$ & $78.0 \pm 27.0, \mathrm{n}=46$ \\
\hline
\end{tabular}

Table 3. The vital sign and BIS index.

\begin{tabular}{ccccccc}
\hline $\begin{array}{c}\text { Time } \\
(\mathrm{min})\end{array}$ & 0 & 10 & 20 & 30 & 40 & $\begin{array}{c}\text { End of } \\
\text { procedure }\end{array}$ \\
\hline $\mathrm{SBP}$ & $123.2 \pm 15.5$ & $126.0 \pm 16.6$ & $120.0 \pm 15.0$ & $113.8 \pm 14.9 *$ & $112.5 \pm 14.6 * 112.2 \pm 12.7 *$ \\
$\mathrm{DBP}$ & $72.3 \pm 8.2$ & $73.5 \pm 10.5$ & $70.8 \pm 11.3$ & $70.0 \pm 9.8$ & $67.5 \pm 11.7$ & $66.1 \pm 8.7 *$ \\
$\mathrm{HR}$ & $68.7 \pm 14.9$ & $55.9 \pm 9.0 *$ & $59.1 \pm 9.3 *$ & $59.4 \pm 8.0 *$ & $59.6 \pm 8.0 *$ & $58.3 \pm 7.5 *$ \\
$\mathrm{BIS}$ & $96.5 \pm 4.4$ & $74.0 \pm 11.2 *$ & $65.3 \pm 12.3 *$ & $65.2 \pm 11.8 *$ & $66.5 \pm 12.3 *$ & $94.2 \pm 5.1$ \\
\hline
\end{tabular}

Values are expressed as mean $\pm \mathrm{SD}$; SBP, systolic blood pressure $(\mathrm{mmHg})$; DBP, diastolic blood pressure $(\mathrm{mmHg})$; HR, heart rate (per minute); BIS, bispectral index; ${ }^{*}, P<0.05$ vs 0 minute in same row, repeated measures analysis of variance

\section{DISCUSSION}

To our knowledge, this is the first report on applying deep sedation in VABB. We believe that applying MAC is effective in patients who are relatively young and in anxious patients and that it increases their satisfaction

VABB was successfully performed with MAC using dexmedetomidine to sedate patients with no significant complications, and there was no respiratory depression or hemodynamic instability.

Since its introduction in the early 2000s, VABB has revolutionized breast cancer diagnosis and has become a widespread tool and a contributor to therapeutic breast conservation. The most common indication was palpable or nonpalpable
American College of Radiology BI-RADS (Breast Imaging Reporting and Database System) lesions in categories 3 and $4 .^{12}$ Recently, B3 lesion management with VABB (including flat epithelial atypia, papillary lesions, radial scars with atypia, benign phyllodes tumors, and low-grade forms of lobular neoplasia) was recommended instead of surgical excision. ${ }^{13}$ The paradigm has shifted in favor of $\mathrm{VABB}$ as a new minimally invasive excision tool for benign and atypical breast lesions. ${ }^{14}$

VABB needles have different diameters: 8G, $11 \mathrm{G}$, and $14 \mathrm{G}$. We used the $8 \mathrm{G}$ needle, which can collect 250 to $310 \mathrm{mg}$ of tissue (three times more than $11 \mathrm{G}$ needles), so that it can resect breast lesions smaller than $3 \mathrm{~cm}$ as well as larger ones. ${ }^{12}$ However, larger needles are associated with pro- 
cedural pain. Pain is an important adverse outcome of VABB; In addition, Zagouri et al. reported maximum pain VAS scores of more than 6 , and patients required second doses of lidocaine. ${ }^{3}$

Proper sedation as well as local anesthesia are needed to reduce pain and patient stress. With regard to immobility based on needle sharpness or size, deep sedation is more reasonable than light sedation; unexpected movement during VABB is dangerous because the needles are sharp and thick. However, general anesthesia does not take advantage of VABB and increases the cost of medical care; therefore, MAC can be an alternative for deep sedation during VABB instead of local and general anesthesia. In our patients, the BIS scores were low enough to indicate deep sedation.

However, during deep sedation, respiratory depression is always a problem. Therefore, the authors have used dexmedetomidine that is known to have minimal respiratory depressive effects. ${ }^{10}$ Because dexmedetomidine was used as the main anesthetic in our study, there was no reduction in the respiratory drive that would require advanced airway interventions. Further, it has analgesic and anxiolytic properties and is well tolerated in different age groups across a broad range of surgical and diagnostic procedures. Dexmedetomidine achieves target sedation levels, which is safe and increases patient satisfaction. Patients can be easily awakened just by talking to them, and BIS safely returns to awake levels. ${ }^{9}$ All procedures were successful, and no patient awoke during surgery. However, an average of 17 \pm 5.2 minutes was required until the start of the procedure, and there was an average recovery time of $20.1 \pm 10.3$ minutes after the operation. The onset time of dexmedetomidine was relatively long compared to the mean operation time (50.1 \pm 24.9 minutes), and this was attributable to prolonged dexmedetomidine onset time. ${ }^{11}$ The mean time from the completion of the dexmedetomidine injection to the time of alertness was 20.1 \pm 10.3 minutes due to the slow offset feature of dexmedetomidine. To decrease the procedure time, it is necessary to reduce the onset time or the recovery time which is possible with the administration of additional drugs, such as midazolam or fentanyl, and by reducing the dexmedetomidine dose. In our study, patients who received a high dose of midazolam or fentanyl showed an earlier onset of sedation with a lower dose of dexmedetomidine without any adverse events.

The purpose of this study was to collect and report data on sedating patients with MAC during VABB. However, there were limitations. First, this was a retrospective chart analysis; hence we were not able to compare the satisfaction of VABB patients or practitioners who did not perform MAC. Second, dexmedetomidine does not cause respiratory problems, but it takes longer to sedate. Therefore, further research is needed on how and by how much sedation time can be reduced.

VABB is widely used for its multiple advantages. VABB under dexmedetomidine MAC can reduce the stress and pain of the patient. Although there are disadvantages of increased induction time, 
appropriate sedation for patient comfort is a more important consideration.

\section{REFERENCES}

1. Ding B, Chen D, Li X, Zhang H, Zhao Y. Meta analysis of efficacy and safety between Mammotome vacuum-assisted breast biopsy and open excision for benign breast tumor. Gland Surg 2013;2:69-79.

2. Hoorntje LE, Peeters PH, Mali WP, Borel Rinkes IH. Vacuum-assisted breast biopsy: a critical review. Eur J Cancer 2003;39:1676-83.

3. Zagouri F, Sergentanis TN, Gounaris A, Koulocheri D, Nonni A, Domeyer P, et al. Pain in different methods of breast biopsy: emphasis on vacuum-assisted breast biopsy. Breast 2008;17:71-5.

4. Zografos GC, Zagouri F, Sergentanis TN, Nonni A, Domeyer P, Koulocheri D, et al. Pain during vacuum-assisted breast biopsy: are there any predictors? Breast 2008;17:592-5.

5. Eller A, Janka R, Lux M, Saake M, Schulz-Wendtland R, Uder M, et al. Stereotactic vacuum-assisted breast biopsy (VABB)--a patients' survey. Anticancer Res 2014;34:3831-7.

6. Gounaris A, Zagouri F, Sergentanis TN, Provatopoulou X, Kalogera E, Sagkriotis A, et al. Vacuum-assisted Breast Biopsy: Insight into Stress-induced Endocrine Events. In Vivo 2007;21:1081-4.

7. ASA: Position on monitored anesthesia care. [Cited 2013 Oct 16]. Available from: http://www.asahq.org/ /media/sites/asahq/

files/public/resources/standards-guidelines/position-on-monitored-anesthesia-care/.

8. Rasheed MA, Punera DC, Bano M, Palaria U, Tyagi A, Sharma S. A study to compare the overall effectiveness between midazolam and dexmedetomidine during monitored anesthesia care: A randomized prospective study. Anesth Essays Res 2015;9:167-72.

9. Hall JE, Uhrich TD, Barney JA, Arain SR, Ebert TJ. Sedative, amnestic, and analgesic properties of small-dose dexmedetomidine infusions. Anesth Analg 2000;90:699-705.

10. Surana P, Parikh DA, Patkar GA, Tendolkar BA. A prospective randomized controlled double-blind trial to assess the effects of dexmedetomidine during cleft palate surgery. Korean J Anesthesiol 2017;70:633-41.

11. Arain SR, Ebert TJ. The efficacy, side effects, and recovery characteristics of dexmedetomidine versus propofol when used for intraoperative sedation. Anesth Analg 2002;95:461-6.

12. Park HL, Hong J. Vacuum-assisted breast biopsy for breast cancer. Gland Surg 2014;3:120-7.

13. Rageth CJ, O’Flynn EA, Comstock C, Kurtz C, Kubik R, Madjar H, et al. First International Consensus Conference on lesions of uncertain malignant potential in the breast (B3 lesions). Breast Cancer Res Treat 2016;159:203-13.

14. Bennett IC. The Changing Role of Vacuum-assisted Biopsy of the Breast: A New Prototype of Minimally Invasive Breast Surgery. Clin Breast Cancer 2017;17:323-5. 